\title{
Facteurs de croissance et prolifération du muscle lisse vasculaire dans I'hypertension et le diabète
}

L'excès du potentiel prolifératif des cellules musculaires lisses des vaisseaux sanguins pourrait constituer l'une des anomalies essentielles responsables du développement de l'athérosclérose au cours de l'hypertension artérielle et du diabète. Les mécanismes de cette perturbation semblent être à la fois intrinsèques (hyperréactivité aux facteurs de croissance et diminution de l'inhibition de contact) et extrinsèques (augmentation de l'activité inductrice de prolifération libérée par les plaquettes sanguines). Un traitement adapté du diabète par l'insuline et certains médicaments hypotenseurs peuvent corriger cet excès d'activité des plaquettes sur la multiplication des cellules de la musculeuse des vaisseaux.

\section{Pavel Hamet \\ Vratislav Hadrava Ursula Kruppa Johanne Tremblay}

\section{ADRESSE}

P. Hamet : docteur en médecine, docteur ès science. V. Hadrava: docteur en médecine. U. Kruppa : Master of sciences. J. Tremblay : docteur ès sciences. Institut de recherches cliniques de Montréal, 110, avenue des Pins-

Ouest, Montréal, Québec H2W 1R7, Canada.

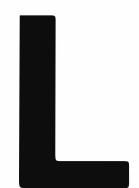

'augmentation de la résistance périphérique vasculaire est l'un des principaux éléments pathogéniques de l'hypertension artérielle (HTA). Cette augmentation de résistance vasculaire s'explique en partie par l'hyperréactivité des vaisseaux [1], mais aussi par une diminution de leur calibre, secondaire à une augmentation du nombre de cellules musculaires lisses de leur paroi [2-4]. Cette hypothèse, initialement suggérée par Folkow [3, 4], a été récemment vérifiée aussi bien dans des modèles animaux que chez des patients souffrant d'hypertension. Nous discuterons ici des différents facteurs qui contribuent à l'augmentation de la prolifération des cellules du muscle lisse vasculaire (VSMC, vascular smooth muscle cells). Nous traiterons plus particulièrement des anomalies intrinsèques de croissance des VSMC et de la contribution potentielle des plaquettes sanguines à la prolifération accrue de ces cellules dans l'hypertension et le diabète.

\section{Rôle primaire de l'augmentation de la prolifération des VSMC dans la pathogénie de I'HTA}

L'étude de la physiopathologie de l'HTA impose une distinction entre les événements à l'origine de la maladie et ceux qui résultent de l'augmentation de la pression artérielle. Une série de critères ont été proposés pour identifier ces événements dits primaires [5, 6]. L'un d'entre eux est naturellement l'antériorité de mise en évidence d'un paramètre par rapport à l'installation de l'hypertension proprement dite. Par exemple, l'hyperplasie du cour et des reins 
survient à un stade précoce dans différents modèles animaux d'hypertension spontanée (d'origine génétique), tels les rats de souche SHR [7], de Nouvelle-Zélande et de Lyon [8]. L'incorporation in vivo de ${ }^{3} \mathrm{H}$-thymidine dans l'ADN nouvellement synthétisé est plus élevée dans le cour, le rein et l'aorte de rats nouveau-nés SHR que chez les rats normotendus Wistar-Kyoto (WKY) [9]. Un deuxième critère est la persistance du facteur étiologique possible dans les cellules mises en culture. De ce point de vue, les cellules du muscle lisse d'aorte de rats SHR continuent de montrer des anomalies de croissance même après des périodes prolongées de culture in vitro [10-15]. L'accroissement de prolifération des

\section{*ABRÉVIATIONS*}

DME : Dulbecco's Modified Eagle Medium (Gibco Laboratories, Burlington, Ontario, Canada).

$\boldsymbol{E} \boldsymbol{G} \boldsymbol{F}$ : epidermal growth factor, facteur de croissance épidermique. ITS : insuline, $2,5 \mu \mathrm{g} / \mathrm{ml}$; transferrine, $2,5 \mathrm{\mu g} / \mathrm{ml}$; sélénium, $2,5 \mathrm{ng} / \mathrm{ml}$.

$\boldsymbol{P D G F}$ : platelet derived growth factor, facteur de croissance dérivé des plaquettes.

SHR : rats spontanément hypertendus de souche Okamoto.

TGF : transforming growth factor, facteur de croissance transformant.

VSMC : vascular smooth muscle cells, cellules $d u$ muscle lisse vasculaire.

WKY : souche de rats normotendus Wistar-Kyoto.

cellules du muscle lisse d'aorte de rats SHR constitue un phénotype stable de la troisième à la vingtième génération et est déjà visible au stade initial d'hypertension puisqu'il a été observé chez des rats âgés de quatre semaines [16]. Il semble entièrement dépendant de la présence des facteurs de croissance [15] et non pas de différences d'efficacité d'attachement initial entre les deux souches de rats SHR et WKY. Comme le montrent aussi bien le décompte du nombre des cellules [15] que la mesure d'incorporation de ${ }^{3} \mathrm{H}$-thymidine dans $\mathrm{m} / \mathrm{s} n^{\circ} 9$ vol. 5, novembre 89

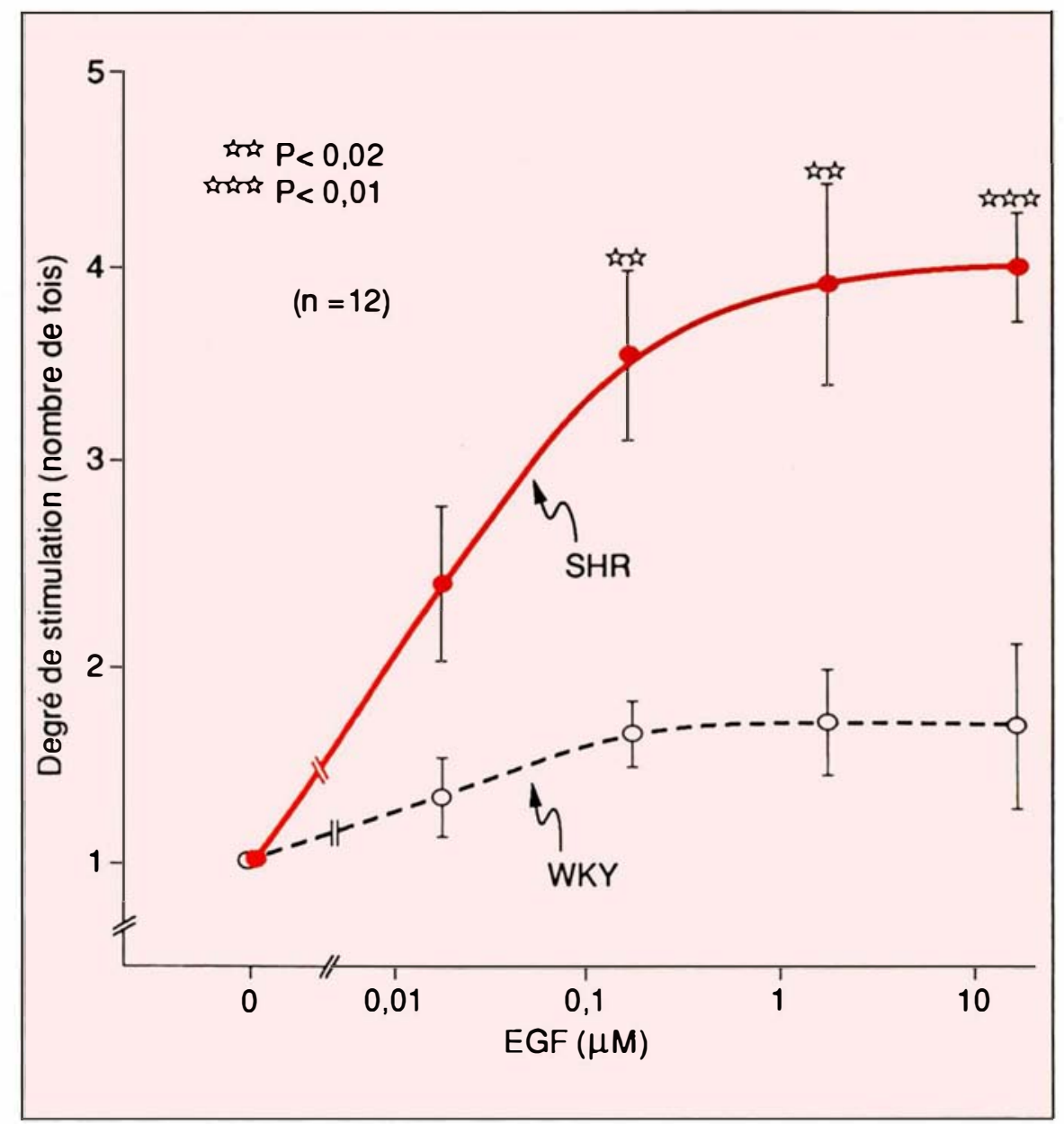

Figure 1. Stimulation de l'incorporation de ${ }^{3}$ H-thymidine dans I'ADN des cellules aortiques de muscle lisse vasculaire du rat par le facteur de croissance épidermique (EGF) (moyenne \pm écart type). SHR, rats spontanément hypertendus. ${ }^{* *} p<0,02,{ }^{* * *} p<0,01$ tel que comparé aux rats WistarKyoto (WKY) par le test $t$ de Student; $p<0,001$, tel qu'évalué par I'analyse de variance. (D'après [15].)

l'ADN, les cellules musculaires lisses de rats SHR répondent exagérément aux facteurs de croissance contenus dans le sérum de veau, facteur de croissance épidermique (EGF) (figure 1) et facteur de croissance dérivé des plaquettes (PDGF).

La prolifération anormale des VSMC est à l'origine de la formation des plaques athérosclérotiques et il a été proposé que le PDGF soit l'un des facteurs initialement responsables de cet épaississement focal de la paroi de l'intima [17]. Bien que les mitogènes plaquettaires y jouent certainement un rôle important, cette prolifération peut débuter avant la dénudation endothéliale et la présence des plaquettes dans l'intima, puisque les VSMC retrouvées dans les plaques athérosclérotiques sécrètent elles-mêmes une activité mitotique de type PDGF et expriment le gène de PDGF-A[18]. L'activité mitotique de l'interleukine l (IL-l) sur les VSMC est modulée par la production de PDGF-A [19]. La régulation autocrine et/ou paracrine des effets mitotiques sur le muscle lisse vasculaire pourrait donc être altérée dans l'hypertension et l'athérosclérose.

Dans l'hypertension, il semble que la différence de croissance entre les cellules de rats normotendus WKY et hypertendus SHR soit la plus marquée au moment où les cellules approchent de la confluence, ce qui 
Tableau I

EFFET DE LA DENSITÉ CELLULAIRE SUR LE TAUX DE CROISSANCE SPÉCIFIQUE DES CELLULES DU MUSCLE LISSE VASCULAIRE

\section{RÉFÉRENCES}

1. Gray SD, DeMey JG. Vascular reactivity in neonatal spontaneously hypertensive rats. Prog Appl Microcirc $1985 ; 8$ : 173-80.

2. Mulvany MJ, Hansen PK, Aalkjaer C. Direct evidence that the greater contractility o: resistance vessels in spontaneously hypertensive rats is associated with narrowed lumen, thickened media and an increased number of smooth muscle cell layers. Circ Res $1978 ; 43: 854-64$

3. Folkow B. Constriction-distention relationships of resistance vessels in normo- and hypertension. Clin Sci 1979; 57 : 23s-5.

4. Folkow B. Physiologic aspects of primary hypertension. Physiol Rev $1982 ; 62$ : 347-503.

5. Hamet P, Tremblay J, Pang SC, Walter SV Wen YI. Primary versus secondary events in hypertension. Can J Physiol Pharmacol 1985 ; $63: 380-6$.

6. Hamet $\mathrm{P}$, Tremblay J. Cyclic nucleotides in the pathogenesis of hypertension. In : Laragh JH, Brenner BM, eds. Hypertension Pathophysiology, Diagnosis and Manage ment. New York: Raven Press, 1990 (sous presse).

7. Kunes J, Pang SC, Cantin M, Genest J, Hamet P. Cardiac and renal hyperplasia in newborn spontaneously hypertensive rats. Clin Sci 1987; 72 : 271-5

8. Pang SC, Long C, Poirier M, et al. Cardiac and renal hyperplasia in newborn genetically hypertensive rats. J Hypertens 1986; 4 (suppl. 3) : S119-22

9. Walter SV, Hamet P. Enhanced DNA synthesis in heart and kidney of newborn spontaneously hypertensive rats. Hypertension $1986 ; 8: 520-5$.

10. Yamori $Y$, Igawa $T$, Kanbe $T$, Kihara $M$, Nara Y, Horie R. Mechanisms of structural vascular changes in genetic hypertension: analyses on cultured vascular smooth muscle cells from spontaneously hypertensive rats. Clin Sci 1981 ; 61 : $121 \mathrm{~s}-3$.

11. Hamet P, Hadrava V, Kruppa U, Trem blay J. Vascular smooth muscle cell hyperresponsiveness to growth factors in hypertension. J Hypertens 1988; 6 (suppl. 4) : S36-9.

12. Blennerhassett MG, Kannan MS, Garfield RE. Density-dependent hyperpolarization in cultured aortic smooth muscle cells. Am J Physiol 1989; 256 : C644-51.

13. Paquet JL, Baudouin-Legros $M$, Marche P, Meyer P. Enhanced proliferating activity of cultured smooth muscle cells from SHR. Am J Hypertens 1989; 2 : 108-10.

\begin{tabular}{|c|c|c|c|}
\hline \multicolumn{4}{|c|}{ Tableau I } \\
$\begin{array}{c}\text { EFFET DE LA DENSITÉ CELLULAIRE SUR LE TAUX DE CROISSANCE } \\
\text { SPÉCIFIQUE DES CELLULES DU MUSCLE LISSE VASCULAIRE }\end{array}$ \\
\hline \multicolumn{3}{|c|}{ Taux de croissance spécifique (jour - $\left.{ }^{-1}\right)$} \\
\hline $\begin{array}{c}\text { Densité cellulaire } \\
\text { a l'inoculation } \\
\text { (nombre de cellules } \\
\times 10^{5} \times \text { plaque - } 1 \text { ) }\end{array}$ & WKY & SHR & $\begin{array}{c}\text { Différence (\%) } \\
\text { (SHR contre WKY) }\end{array}$ \\
\hline 2,0 & $0,73 \pm 0,12$ & $0,88 \pm 0,16$ & $121 \pm 21$ \\
\hline 4,0 & $0,36 \pm 0,05$ & $0,56 \pm 0,02^{*}$ & $156 \pm 14$ \\
\hline 10,0 & $0,03 \pm 0,01$ & $0,19 \pm 0,02^{* *}$ & $630 \pm 140$ \\
\hline
\end{tabular}

Les données sont exprimées en moyenne \pm écart type. WKY: rats Wistar-Kyoto ; SHR : rats spontanément hypertendus. Le taux de croissance spécifique est déterminé selon la formule $\left(P_{2}-P_{1}\right) / P_{1}\left(t_{2}-t_{1}\right)$, où $P_{1}$ et $P_{2}$ représentent la densité cellulaire d̀ $t_{1}$ (jour de l'inoculation) $e$ $t_{2}$ (trois jours après l'inoculation). ${ }^{*} p<0,02,{ }^{*} p<0,01$ par rapport aux rats WKY en utilisant le test $t$ de Student. (D'après [15].) indique un défaut de l'inhibition par contact (Tableau I). Il a été suggéré [20] qu'une hyperproduction de la matrice extracellulaire puisse être responsable de cette augmentation de croissance.

Le facteur de croissance transformant $\beta$ (TGF $\beta$ ) est connu pour son rôle dans l'accumulation des protéines au niveau de la matrice extracellulaire [21] ainsi que la modulation de la croissance cellulaire. Il inhibe la prolifération des VSMC normales à faible densité, alors qu'il l'augmente quand les cellules approchent la confluence [22]. Le PDGF et l'EGF stimulent l'expression de TGF $\beta$ qui possède lui-même la capacité d'autocontrôler sa propre expression [23]. Des interactions complexes entre différents types de facteurs de croissance incluant le PDGF et l'EGF sont impliquées dans les actions biologiques de TGF $\beta$ [24]. Le fait que le taux d'expression des ARNm du TGF $\beta$ soit plus élevé dans les aortes de rats hypertendus sous l'effet d'un régime $\mathrm{DOCA}^{*} /$ sel [25] souligne

* DOCA : deoxycorticosterone acetate (acétate de désoxycorticostérone). aussi le rôle potentiel du TGF $\beta$ sur l'hyperplasie des cellules du muscle lisse vasculaire

Dans le but d'identifier les mécanismes moléculaires menant à l'hyperplasie du muscle lisse vasculaire dans l'hypertension, nous avons récemment étudié l'expression des oncogènes cellulaires impliqués dans la transition de la phase $G_{0}-G_{1}$ à la phase $S$ du cycle cellulaire. C'est à ce point de transition que les cellules normales ont besoin de facteurs de croissance tels le PDGF, facteur de compétence, et l'EGF, facteur de progression. Ces deux facteurs de croissance stimulent l'expression des proto-oncogènes $c$-myc et $c$-fos [2628]. Des analyses par Northern blot, après stimulation par le sérum de veau, ont montré une augmentation plus importante d'expression de ces proto-oncogènes $c$-fos et $c$-myc dans les VSMC de rats SHR que dans les cellules de rats WKY [11].

Récemment, nous avons entrepris d'étudier l'expression du TGF $\beta$ dans ces mêmes cellules. Nos résultats préliminaires montrent que le TGF $\beta$ est exprimé dans les VSMC et de façon plus abondante dans les cellules en croissance que dans les cellules quiescentes (figure 2). Le contrôle de 
l'expression de TGF $\beta$ dans les VSMC de rats SHR et WKY est en cours d'étude dans notre laboratoire.

\section{Effet des plaquettes sur la croissance du muscle lisse vasculaire dans I'HTA et le diabète}

Ross et al. [29] ont montré, il y a déjà une dizaine d'années, que des cellules synchronisées dans la phase $G_{0}$ par une restriction des facteurs de croissance deviennent sensibles à l'addition d'extraits plaquettaires. Depuis, il a été démontré que les plaquettes transportent plusieurs facteurs de croissance, comme le PDGF, l'EGF et le TGF $\beta$ [29-31]. Le métabolisme du glucose et sa régulation homéostatique, dont dépend la prolifération cellulaire, sont contrôlés à plusieurs niveaux. Au niveau de la membrane cellulaire, le transport du glucose s'effectue via un mécanisme de diffusion facilitée qui dépend d'un transporteur de glucose. L'expression plus élevée de ce transporteur associée à une augmentation du transport de glucose a été observée dans les cellules transformées par les oncogènes $v$-src et $c$-ras [32]. L'expression de l'un de ces transporteurs est contrôlée par le PDGF [30, 33]. La stimulation par le PDGF n'augmente pas seulement l'expression de l'un des transporteurs de glucose, mais aussi son association à la membrane et l'activité du transport de glucose [30]. Les plaquettes semblent aussi constituer un réservoir physiologique majeur de TGF $\beta$ puisqu'elles contiennent de 40 à 100 fois plus de ce facteur que tout autre tissu non néoplasique étudié à ce jour [34]. Cette observation constitue un argument supplémentaire en faveur du rôle physiologique important du TGF $\beta$ dans la réparation et la régénération tissulaire ainsi que la prolifération des cellules du muscle lisse vasculaire. Même si le TGF $\beta$ seul ne constitue pas un mitogène très puissant, son action proliférative augmente rapidement en présence de l'EGF [35].

\section{Plaquettes de diabétiques insulino-dépendants}

Les plaquettes provenant de diabétiques insulino-dépendants possèdent $\mathrm{m} / \mathrm{s} n^{\circ} 9$ vol. 5 , novembre 89

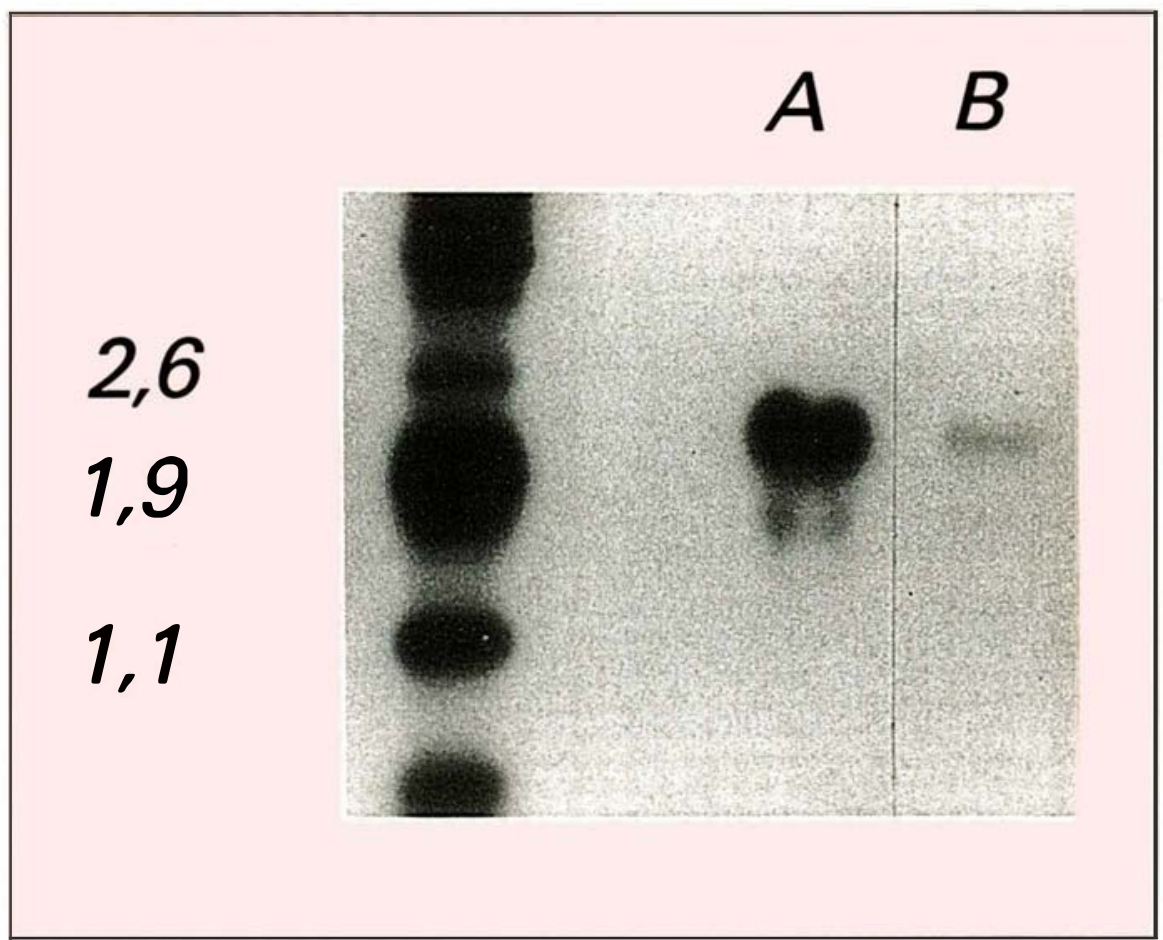

Figure 2. Analyse de l'expression de TGF $\beta$ par Northern blot dans les cellules VSMC de rat en culture stimulées par $10 \%$ de sérum de veau (A) et en état de quiescence (B). La quiescence des cellules a été établie avec un milieu DME et ITS pendant 50 heures. La taille moléculaire du messager TGF $\beta$ est de 2,4 kb; $10 \mu \mathrm{g}$ d'ARN total ont été déposés dans chaque puits. Le gel a été coloré au bromure d'éthidium pour s'assurer de la quantification des échantillons d'ARN. La membrane a été hybridée avec une sonde d'ADNc de TGFB [46] marquée au 32 P par la méthode de l'amorçage au hasard.

une activité proliférative accrue sur les cellules du muscle lisse vasculaire [36, 37]. Cet effet prolifératif accru pourrait être dû à: (1) une anomalie intrinsèque des facteurs de croissance dans le diabète; (2) une augmentation du contenu en facteur(s) de croissance des plaquettes de diabétiques ; (3) un effet secondaire à des changements dans l'intégrité de la couche endothéliale de la paroi vasculaire rendant le muscle lisse vasculaire plus exposé aux plaquettes pouvant conduire à : (4) une longévité altérée des plaquettes de diabétiques. Il est en effet connu que les plaquettes de patients diabétiques ont une longévité réduite [38]. Il est donc possible que, par suite d'une consommation périphérique accrue des plaquettes, celles-ci soient plus jeunes chez les patients diabétiques, ce qui leur conférerait un potentiel de croissance plus élevé. En effet, le contenu en granules $\alpha$, site de stockage d'EGF et de PDGF, est plus élevé dans les plaquettes jeunes [39]. Ce paramètre mérite d'être évalué dans le diabète par les mesures directes du contenu plaquettaire en facteur(s) de croissance. Bien que les mécanismes cellulaires de cette augmentation d'activité proliférative des plaquettes soient encore mystérieux, on peut prévoir des conséquences cliniques importantes pour une telle anomalie. Ainsi, chez les patients diabétiques insulino-dépendants, une augmentation de l'activité proliférative des plaquettes pourrait conduire à une augmentation de croissance du muscle lisse vasculaire et, par conséquent, au développement de macroangiopathie et de complications cardiovasculaires souvent observées dans cette maladie. 
14. Scott-Burden T, Resink TJ, Baur U, Burgin M, Buhler FR. Response to epiderma growth factor by cultured smooth muscle cells from hypertensive rats. Hypertension 1989 ; 13 : 295-304.

15. Hadrava V, Tremblay J, Hamet P. Abnormalities in growth characteristics of aortic smooth muscle cells in spontaneously hypertensive rats. Hypertension 1989 ; 13 : 589-97.

16. Berk BC, Vallega G, Muslin AJ, Gordon HM, Canessa M, Alexander RW. Spontaneously hypertensive rat vascular smooth muscle cells in culture exhibit increased growth and $\mathrm{Na}+/ \mathrm{H}^{+}$exchange. J Clin Invest $1989 ; 83: 822-9$.

17. Ross R, Harker L. Hyperlipidemia and atherosclerosis. Chronic hyperlipidemia initiates and maintains lesions by endothelial cell desquamation and lipid accumulation. Science 1976 ; 193 : 1094-100.

18. Libby P, Warner SJC, Salomon RN, Birinyi LK. Production of platelet-derived growth factor-like mitogen by smooth-muscle cells from human atheroma. N Engl J Med 1988 ; 318 : 1493-8.

19. Raines EW, Dower SK, Ross R. Interleukin-l mitogenic activity for fibroblasts and smooth muscle cells is due to PDGF-AA. Science 1989 ; 243 : 393-6.

20. Scott-Burden T, Resink TJ, Burgin $M$, Buhler FR. Extracellular matrix : differential influence on growth and biosynthesis patterns of vascular smooth muscle cells from SHR and WKY rats. J Cell Physiol 1989 (sous presse).

21. Penttinen RP, Kobayashi S, Bornstein P. Transforming growth factor $\beta$ increases mRNA for matrix proteins both in the presence and in the absence of changes in mRNA stability. Proc Natl Acad Sci USA 1988; 85 : 1105-8.

22. Majack RA. Beta-type transforming growth factor specifies organizational behavior in vascular smooth muscle cell cultures. J Cell Biol 1987 ; 105 : 465-71.

23. Van Obberghen-Schilling E, Roche NS, Flanders KC, Sporn MB, Roberts AB. Transforming growth factor $\beta 1$ positively regulates its own expression in normal and transformed cells. J Biol Chem 1988; 263 : 7741-6.

24. Sporn MB, Roberts AB, Wakefield LM, Assoian RK. Transforming growth factor- $\beta$ : biological function and chemical structure. Science $1986 ; 233$ : 532-4.

25. Sarzani $\mathbf{R}$, Brecher $\mathbf{P}$, Chobanian AV Growth factor expression in aorta of normotensive and hypertensive rats. J Clin Invest
L'augmentation de l'effet prolifératif des plaquettes de diabétiques peut être normalisée par une insulinothérapie intensive, que ce soit par injections multiples ou à l'aide de pompes à perfusion d'insuline [40, 41]. Dans ce cas, la perfusion d'insuline est accompagnée de bolus d'insuline aux périodes des repas, calculés en fonction de l'ingestion en glucides. Dans le cas du diabète bien contrôlé, la normalisation de la glycémie et de l'hémoglobine glycosylée s'accompagne d'une diminution de la protéinurie et d'une amélioration significative de la neuropathie et du flot sanguin aux extrémités [42]. Il est intéressant de constater que ce traitement conduit à une quasi-nor- malisation du pouvoir prolifératif des plaquettes de ces patients, après cinq mois de traitement, par l'un ou l'autre de ces deux types d'insulinothérapie (figure 3). Bien qu'il n'y ait aucune corrélation significative entre l'activité proliférative des plaquettes et la glycémie, le cholestérol ou l'hémoglobine glycosylée, une telle corrélation positive existe avec la dose d'insuline à action prolongée. En revanche, l'activité proliférative des plaquettes semble évoluer en sens contraire, c'est-à-dire diminuer, à la suite d'administration discontinue d'insuline à courte durée d'action, à l'occasion de chaque repas. Ces études suggèrent donc que l'hyperinsulinémie soutenue est associée

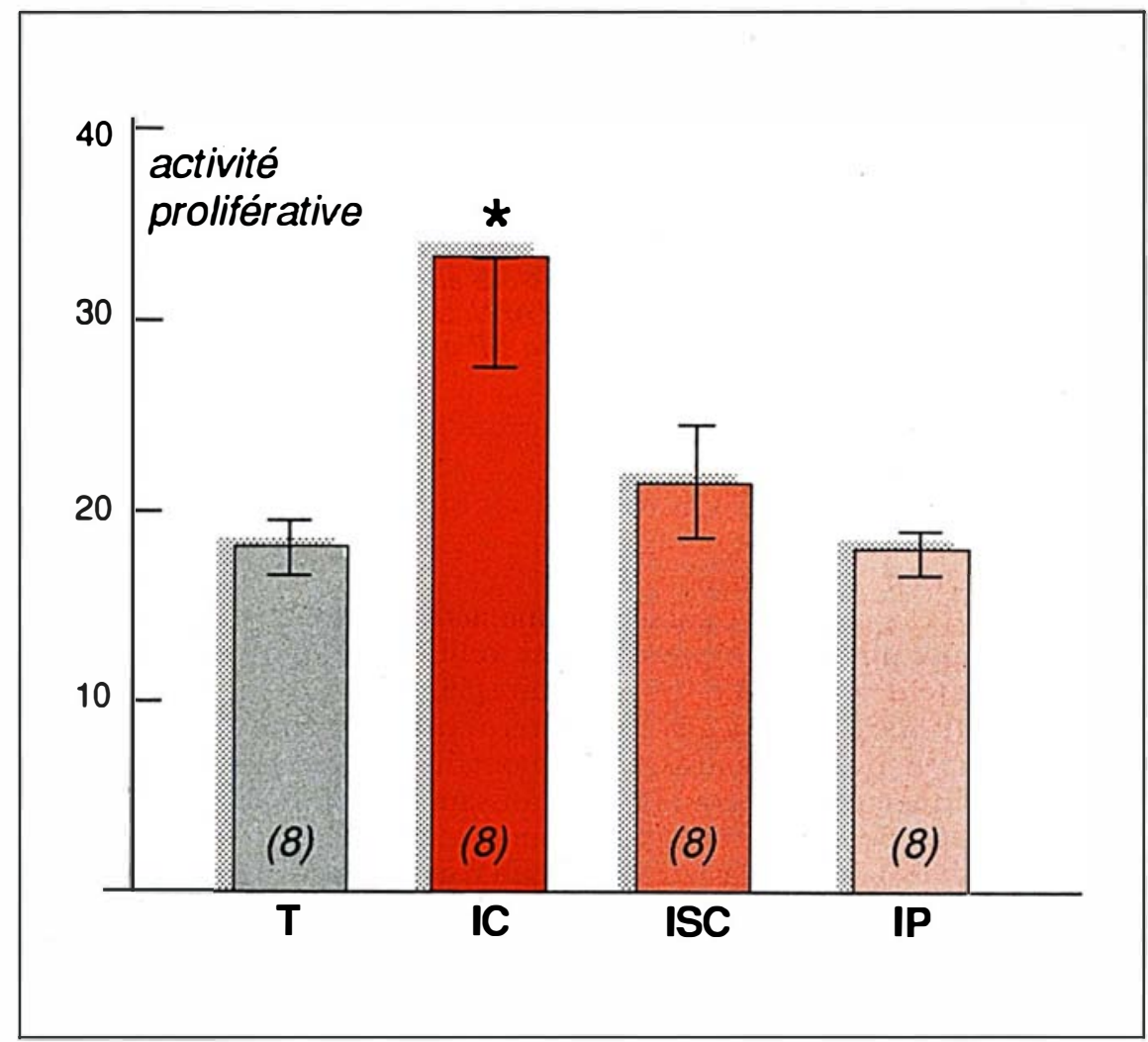

Figure 3. Activité proliférative des extraits plaquettaires, mesurée par l'incorporation de la ${ }^{3} H$-thymidine dans I'ADN de cellules aortiques du muscle lisse vasculaire de rat. L'activité proliférative des extraits plaquettaires est exprimée en pourcentage de l'activité de croissance observée après l'addition de $10 \%$ de sérum de veau. Les données sont exprimées en moyenne \pm écart type et le nombre d'échantillons est indiqué entre parenthèses. $T=$ témoin; $I C=$ insulinothérapie conventionnelle (une à deux injections d'insuline à action prolongée par jour avec ou sans insuline à courte durée d'action); ISC = infusion souscutanée continue d'insuline, par pompe accompagnée d'un bolus d'insuline à chaque repas ; $I P=$ infuseur $* P e n$ » (injections multiples, accompagnées à chaque repas d'une dose d'insuline ultralente). ${ }^{*} p<0,05$, tel qu'évalué par comparaison au témoin par le test $t$ de Student. (D'après [40].) 
à une activité proliférative excessive des plaquettes. Cette hypothèse méritera une réévaluation plus directe de l'effet d'une hyperinsulinémie constante sur la croissance du muscle lisse vasculaire ainsi que de ses conséquences cliniques possibles. Elle pourrait s'avérer d'autant plus importante que l'insulinémie, en soi, est considérée comme un facteur de risque de macroangiopathie chez les diabétiques [43]. De plus, l'hyperinsulinémie, surtout post-prandiale, a été observée par plusieurs groupes au cours de l'hypertension essentielle, même en l'absence du diabète.

\section{Plaquettes d'hypertendus et de diabétiques non insulino-dépendants}

Plus récemment, nous avons démontré une augmentation de la prolifération des VSMC sous l'effet d'extraits plaquettaires provenant de patients atteints de diabète non insulino-dépendant, mais aussi de sujets hypertendus non diabétiques. En accord avec ces observations, l'activité proliférative plaquettaire la plus importante a été retrouvée chez les malades présentant à la fois une hypertension et un diabète [44]. Puisqu'il est possible d'influencer l'activité proliférative par une insulinothérapie intensive chez les diabétiques, il est apparu utile d'évaluer cette propriété plaquettaire chez les patients hypertendus soumis à un traitement antihypertenseur. Les résultats préliminaires de cette étude sont illustrés sur la figure 4. Il est évident que les plaquettes recueillies chez les patients hypertendus diabétiques ayant reçu $50 \mathrm{mg} / \mathrm{j}$ d'hydrochlorothiazide (un sulfamide diurétique) pendant une période moyenne de deux mois contiennent davantage de facteur mitotique que les plaquettes des mêmes patients ayant reçu un placebo. Des études additionnelles ont démontré que d'autres médicaments, tel l'indapamide*,

\footnotetext{
* Indapamide: diurétique; dipyridamole: inhibiteur plaquettaire, vasodilatateur coronarien; nifédipine: inhibiteur de transport de l'ion calcium; nitroglycérine : vasodilatateur, isoprotérénol : sympathicomimétique.

$\mathrm{m} / \mathrm{s} \mathrm{n}^{\circ} 9$ vol. 5 , novembre 89
}

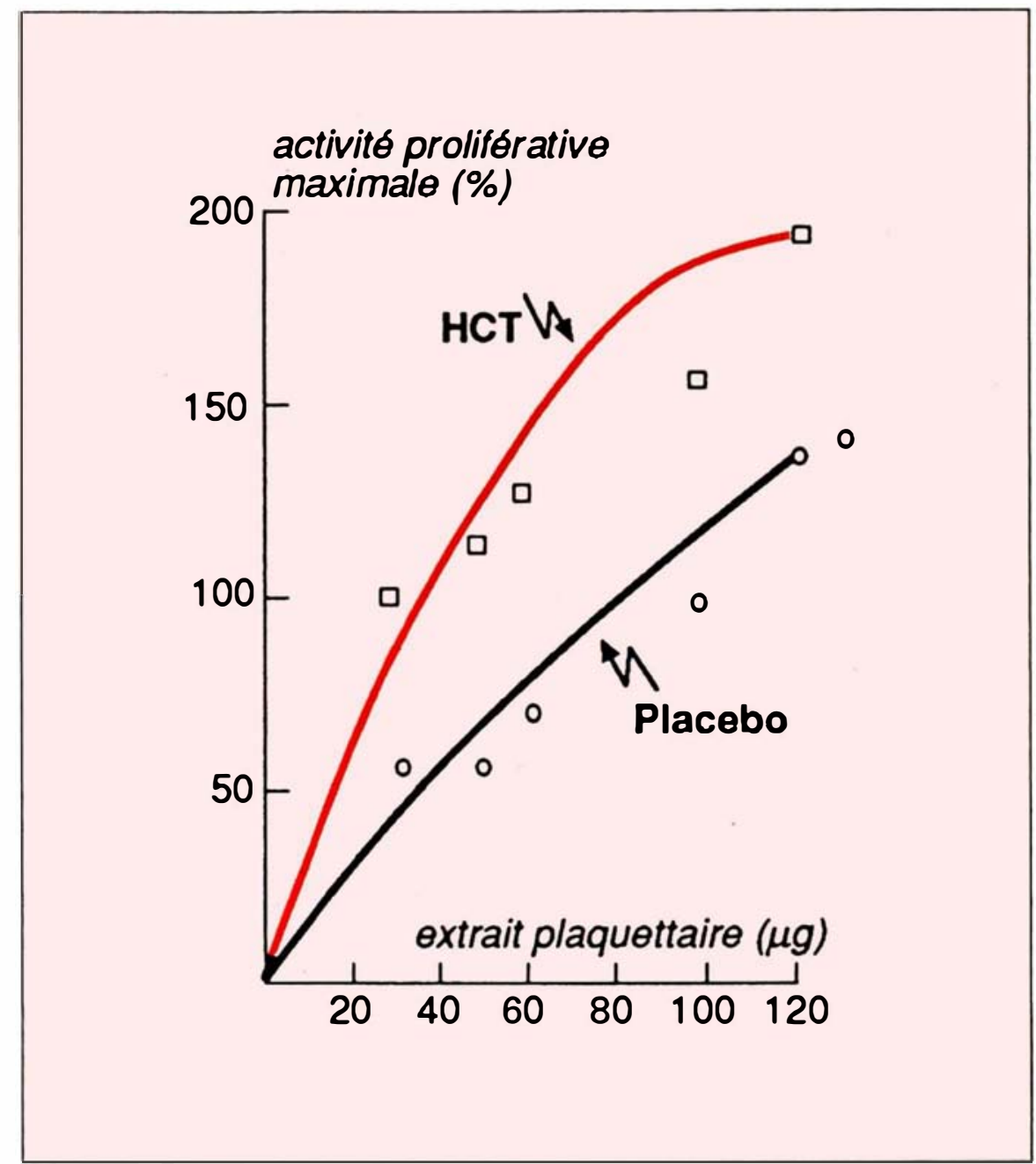

Figure 4. Effet de la concentration croissante d'extraits plaquettaires obtenus chez dix patients hypertendus et diabétiques non insulinodépendants âgés de 40 à 60 ans et recevant un placebo, en comparaison avec l'effet d'extraits obtenus de ces mêmes patients après administration de $50 \mathrm{mg} / \mathrm{j}$ d'hydrochlorothiazide (HCT) pendant huit semaines.

n'induisent pas d'accroissement de cette activité proliférative, ou encore peuvent la diminuer. D'autres médications, incluant les inhibiteurs de l'enzyme de conversion, les inhibiteurs de canaux calciques et les $\beta$ bloquants, sont actuellement à l'étude.

D'autres études ont montré qu'un vasodilatateur coronarien, le trapidil, inhibe la prolifération des cellules BALB/c-3T3 stimulées par le PDGF mais n'a aucun effet sur la stimulation par le fibroblast growth factor (FGF) ou le $\mathrm{Ca}_{3}\left(\mathrm{PO}_{4}\right)_{2}$ ou encore après transformation par le virus oncogène SV-40. Le trapidil présente ainsi une activité anti-PDGF qui n'a pas été observée pour le dipyridamole $^{*}$, la nifédipine ${ }^{*}$, la nitroglycérine $^{*}$, l'isoprotérénol* ou l'aspirine et se révèle être un nouvel outil potentiel pour le traitement de l'athérosclérose et d'autres maladies dans lesquelles la prolifération des cellules du muscle lisse vasculaire et l'activité de PDGF semblent être exagérées [45]. L'activité proliférative plaquettaire la plus élevée se retrouve dans les plaquettes de patients souffrant à la fois d'hypertension et de diabète [44]. Il est donc possible que cette augmentation soit impliquée dans la pathogénie des maladies macrovasculaires et constitue un marqueur utile des désordres de 
croissance du muscle lisse vasculaire. De la même façon, l'effet des différents traitements antihypertenseurs utilisant diverses catégories de médicaments devrait être évalué quant à leur influence sur l'activité proliférative des plaquettes.

\section{Conclusion}

\section{RÉFÉRENCES}

26. Kelly K, Cochran BH, Stiles CD, Leder P. Cell-specific regulation of the $c$-myc gene by lymphocyte mitogens and platelet-derived growth factor. Cell $1983 ; 35: 603-10$.

27. Greenberg ME, Ziff EB. Stimulation of 3T3 cells induces transcription of the $c$-fos proto-oncogene. Nature 1984 ; 311 : 433-8.

28. McCaffrey P, Ran W, Campisi J, Rich Rosner M. Two independent growth factorgenerated signals regulate $c$-fos and $c$-myc mRNA levels in Swiss 3T3 cells. J Biol Chem $1987 ; 262: 1442-5$

29. Ross R, Nist C, Kariya B, Rivest M J, Raines E, Callis J. Physiological quiescence in plasma-derived serum : influence of platelet-derived growth factor on cell growth in culture. J Cell Physiol 1978 ; 97 : 497-508.

30. Rollins B J, Morrison ED, Usher P, Flier JS. Platelet-derived growth factor regulates glucose transporter expression. J Biol Chem 1988 ; 263 : 16523-6.

31. Oka Y, Orth DN. Human plasma epidermal growth factor beta-urogastrone is associated with blood platelets. J Clin Invest 1983 ; 72 : 249-59.

32. Flier JS, Mueckler MM, Usher P, Lodish HF. Elevated levels of glucose transport and transporter messenger RNA are induced by ras or src oncogenes. Science 1987 ; 235 : 1492-5.

33. Mueckler MM, Caruso C, Baldwin SS, et al. Sequence and structure of a human glucose transporter. Science 1985 ; 229 : 941-5.

34. Assoian RK, Komoriya AK, Meyers CA, Miller DM, Sporn MB. Transforming growth factor- $\beta$ in human platelets. J Biol Chem 1983 ; 258 : 7155-60.

35. Childs CB, Proper JA, Tucker RF, Moses HL. Serum contains a platelet-derived transforming growth factor. Proc Natl Acad Sci USA 1982 ; 79 : 5312-6.

36. Hamet $P$, Sugimoto H, Umeda F Franks DJ. Platelets and vascular smooth muscle : abnormalities of phosphodiesterase, aggregation, and cell growth in experimental and human diabetes. Metabolism 1983 ; 32 :
37. Umeda F, Franks DJ, Sugimoto H, Halle JP, Hamet P. Effect of plasma, serum and platelets from diabetics on DNA synthesis in cultured vascular smooth muscle cells. Clin Invest Med 1985 ; 8 : 35-40.

38. Ferguson JC, Mackay N, Philip JAD. Determination of platelet and fibrinogen half-time with ( $\left.{ }^{75} \mathrm{SE}\right)$ selenomethionine : studies in normal and in diabetic subjects. Clin Sci Mol Med 1975 ; 49 : 115-20.

39. Tremblay J, Hamet P. Cyclic nucleotides and calcium in platelets. In : Gordon JL, MacIntyre DE, eds. Platelets in Biology and Pathology, Vol. III. Amsterdam: Elsevier North-Holland Biomedical, 1987 : 433-65.

40. Sugimoto H, Franks DJ, Lecavalier L, Chiasson JL, Hamet P. Therapeutic modulation of growth-promoting activity in platelets form diabetics. Diabetes 1987 ; 36 : 667-72.

41. Chiasson JL, Ducros F, Poliquin. Hamet M, Lopez D, Lecavalier L, Hamet P. Continuous subcutaneous insulin infusion (Mill-Hill Infuser) versus multiple injections (Medi-Jector) in the treatment of insulindependent diabetes mellitus and the effect of metabolic control of microangiopathy. Diabetes Care 1984 ; 7 : $331-7$.

42. Hamet P, Abarca J, Lopez D, et al. Patient self-management of continuous subcutaneous insulin infusion. Diabetes Care 1982 ; $5: 485-91$.

43. Colwell JA. Metabolism Clinical and Experimental, Vol. 34 (suppl. 1). Orlando: Grune and Stratton Inc: 1985.

44. Hamet P, Tremblay J. Abnormalities of second messenger systems in hypertension. In : Meyer P, Marche P, eds. Blood Cells and Arteries in Hypertension and Atherosclerosis (The Argenteuil Symposia Series), Vol. 12. New York : Raven Press, 1989: 171-87.

45. Ohnishi K, Yamaguchi K, Shimada S, Suzuki Y, Kumagai A. A new approach to the treatment of atherosclerosis and trapidil as an antagonist to platelet-derived growth factor. Life Sci 1981; 28 : 1641-6.

46. Derynck R, Jarrett JA, Chen EY, Goeddel DV. The murine transforming growth factor- $\beta$ precursor. J Biol Chem $1986 ; 261: 4377$. 9
La figure 5 schématise l'implication possible des facteurs intrinsèques et extrinsèques dans la régulation de la prolifération du muscle lisse vasculaire. Des facteurs intrinsèques encore inconnus, dont la persistance en culture souligne le caractère primitif dans l'hypertension, sont responsables de la croissance accrue des VSMC en réponse aux facteurs de croissance, tels que le PDGF et l'EGF, celle-ci accompagnée d'une expression accrue des proto-oncogènes. D'autres facteurs intrinsèques ou une expression pléiotropique des mêmes anomalies conduisent à une diminution de l'inhibition par contact de la croissance. Les expériences qui conduiront à la découverte de ces facteurs intrinsèques devront emprunter les outils de la génétique moléculaire. Le défaut majeur des études actuelles est leur restriction au modèle de rats spontanément hypertendus, limitant leur application à l'hypertension essentielle humaine. La coségrégation du locus génétique responsable de l'augmentation de la réponse aux facteurs de croissance avec l'hypertension n'est pas encore démontrée. Si l'augmentation de la prolifération s'avère dépendre de l'un des loci génétiques de l'hypertension, des stratégies de génétique moléculaire devront être entreprises afin d'identifier les gènes responsables de cette anomalie intrinsèque.

Le concours de facteurs extrinsèques à l'augmentation de la prolifération des VSMC a également été discuté dans cet article. La présence d'une activité proliférative accrue dans l'hypertension et le diabète suggère que le contenu plaquettaire en facteurs de croissance est augmenté dans ces désordres. Cependant, dans les plaquettes de sujets hypertendus ou diabétiques, une modification du rapport activateur/inhibiteur de la prolifération des VSMC n'est pas exclue. Plusieurs études soulignent 


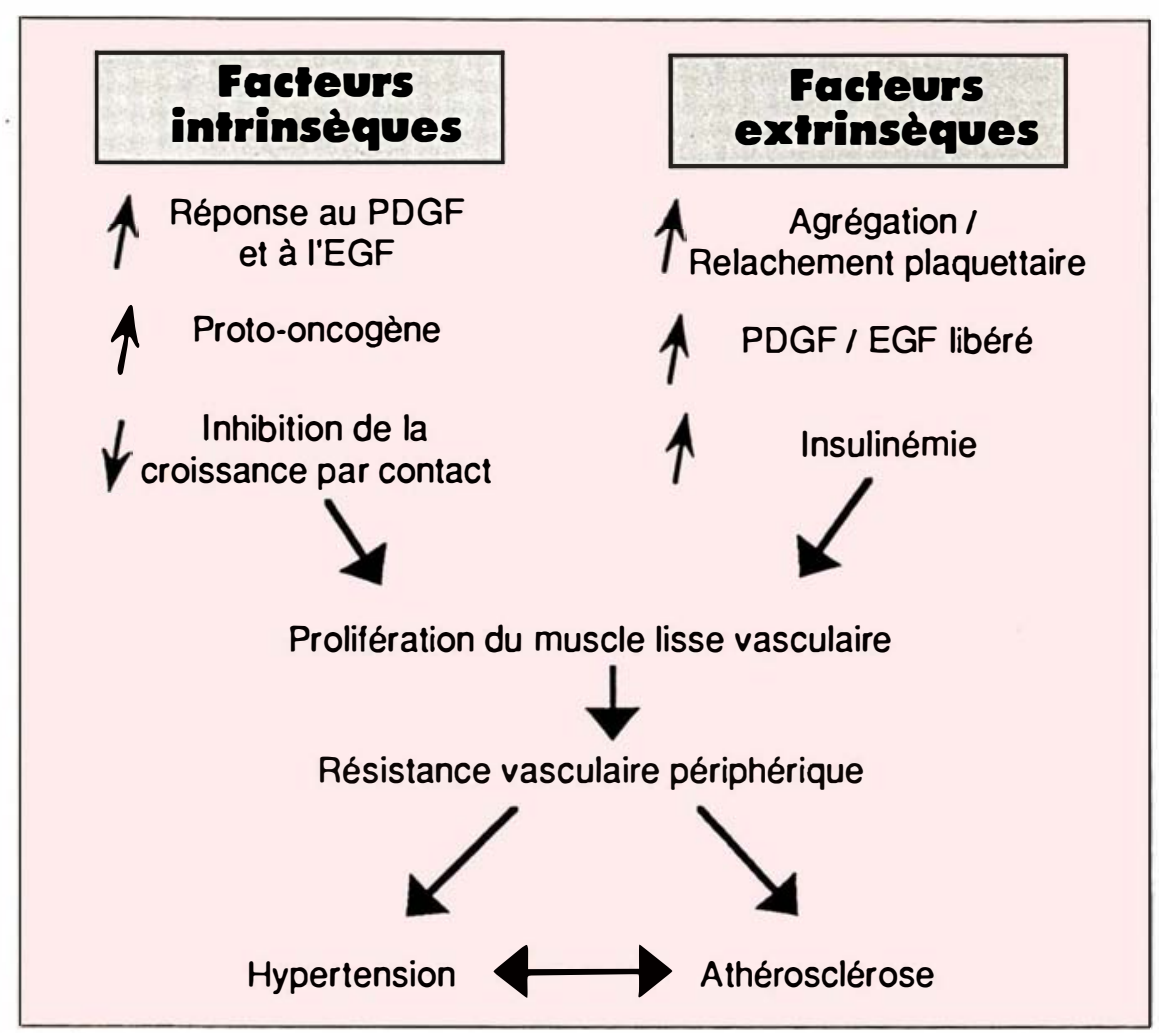

Figure 5. Représentation schématique de l'implication des facteurs intrinsèques et extrinsèques dans la pathogénie des maladies vasculaires.

l'importance de l'hyperinsulinémie comme facteur de risque de développement de maladies vasculaires aussi bien dans l'hypertension que dans le diabète. Dans l'ensemble, les facteurs intrinsèques et extrinsèques contribuent à l'augmentation de la prolifération du muscle lisse vasculaire et leurs rôles respectifs, additifs ou synergiques, nécessitent des études ultérieures.

La prolifération accrue du muscle lisse vasculaire, selon l'hypothèse de Folkow, est le fondement biologique d'une augmentation de la résistance vasculaire périphérique et peut s'accompagner d'une augmentation parallèle de la réponse contractile du vaisseau. Cette anomalie se reflétant au niveau du muscle lisse vasculaire peut ainsi conduire à l'hypertension, peut-être à l'athérosclérose, et contribuer de plus à la coprésentation de ces deux affections majeures responsables des deux tiers de la mortalité de la population des pays industrialisés. L'élucidation des facteurs génétiques et thérapeutiques régularisant l'homéostase de la paroi vasculaire peut ainsi contribuer non seulement à une meilleure compréhension de la pathogénie des maladies cardiovasculaires mais aussi à la prévention de leurs complications

\section{Remerciements}

Ce travail a bénéficié d'un soutien financier du Conseil de recherches médicales du Canada au groupe multidisciplinaire de recherche sur l'hypertension. Vratislav Hadrava et Ursula Kruppa sont respectivement boursiers de The Diabetic Children's Foundation et du Conseil de recherches médicales du Canada ; Johanne Tremblay est chercheur-boursier du Fonds de la recherche en santé du Québec. Les auteurs remercient également le Dr Jacques Viret de ses commentaires judicieux et Mme Louise Chevrefils de son travail de secrétariat. Le Tableau 1 et la figure 1 sont reproduits avec la permission de American Heart Association Inc.

\section{Summary}

Growth factors and proliferation of vascular smooth muscle in hypertension and diabetes

The accelerated atherogenesis of hypertension and diabetes as well as the increased peripheral resistance of hypertension may have, as a common basis, an increased proliferation of the smooth muscle layer of the vessels. Indeed, several studies have recently demonstrated an increased proliferation of vascular smooth muscle cells. Since this increased proliferation persists in culture, even after many passages, it is suggested that it reflects a primary defect in hypertension. The mechanisms of this intrinsic growth defect is yet unknown but it is characterized by a concomitantly increased expression of oncogenes, such as $c$-fos and $c-m y c$, shortening of the $G_{1}$ phase of the cell cycle as well as decrease of contact inhibition and abnormal cellular matrix. In addition to this intrinsic defect, extrinsic factors may contribute to vascular wall abnormalities. Thus, platelets from both diabetic and hypertensive subjects possess an increased proliferative activity on vascular smooth muscle cells. This enhanced proliferative potential of platelets can be normalized by adequate treatment of diabetes and both positively and negatively modulated by antihypertensive therapy. The additive or synergetic effects of these intrinsic and extrinsic factors on the proliferation of vascular smooth muscle cells as well as their consequences for the development of complications of hypertension and diabetes deserve further attention.

\section{TIRÉS A PART}

\section{P. Hamet.}

